\title{
IMPROVED PATH PLANNING METHOD FOR AUTONOMOUS LOADING OPERATION BY WHEEL LOADER
}

\author{
Shigeru Sarata, Noriho Koyachi \\ National Institute of Advanced Industrial Science and Technology (AIST), Tsukuba, Japan \\ Takashi Tsubouchi \\ University of Tsukuba, Tsukuba, Japan
}

\begin{abstract}
Authors have been conducting research on an autonomous system for loading operation by wheel loader. In loading operation with $\mathrm{V}$ shape loading method, a time for travelling is the dominant factor for the cycle time. The path should be modified varied condition of the working place. To achieve quicker and reliable operation in autonomous loading operation, new path planning method is developed. In this method, the path planning is based on Look Ahead control and combination of the path elements. The new developed method generates shorter path with high flexibility compare to the planned path by the older version of path planning in the research. Detail of the method and simulation results will be described in this paper
\end{abstract}

KEYWORDS: path planning, loading operation, autonomous system, wheel loader.

\section{INTRODUCTION}

Authors have been conducting a research on an autonomous system for loading operation by wheel loader. The developed autonomous loading system was demonstrated with real size experimental model: YAMAZUMI No.4(YZ4) [1]. In loading operation, travelling between scooping point and dumping point is dominant factor for cycle time of the operation. In previous version of the developed system in the research, the travelling path for $\mathrm{V}$ shape loading is formed by combination of path elements: straight and curve elements. The planned path is represented by five parameters such as length of curve and straight lines. This method has very simple procedure and simple form representing the planned path however it provides longer path compare to the path in operation by human operator. Also it provides paths with less flexibility. Longer path needs more time for travel and longer cycle time of the operation. To reduce the cycle time and to achieve flexible operation for more efficiency in the autonomous operation, new version of path planning method has been developed. In the new developed method, the path is generated with combination of path elements in the leaving wing and based on Look Ahead control in the approach wing. In following sections, theoretical analysis and example results of the new developed path planning method are described. 


\section{PATH PLANNING WITH PATH ELEMENTS}

In the previous version of path planning, the highest priority is put on easy planning for computer processing. The planned path is formed by a combination of the path elements. The path elements are the straight element and the curve elements. As the curve elements, Symmetrical Pseudo Clothoid (SPC) is defined. Pseudo clothoid is modified clothoid based on steering mechanism of wheel loader. SPC is formed by two same pseudo clothoid connected in reverse direction. At the ends of SPC, curvature is zero, that is, the steering angle is zero. SPC can be connected to other SPC or straight element continuously.

$\mathrm{V}$ shape path consists of a curve part and a straight part. A curve part consists of a sequence of a straight line, SPC and a straight line, in that order. Fig.1 (a) shows the steering angle travelling on a curve part and Fig.(b) shows corresponding path.

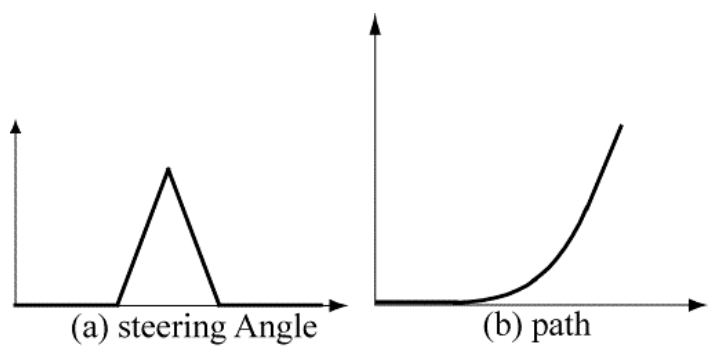

Figure 1: Curve part with Symmetrical Pseudo Clothoid

Because the path following control has higher accuracy in the straight part, the curve part is used in the leave wing of $\mathrm{V}$ shape path and the straight part is used in the approach wing as shown in Fig.2.

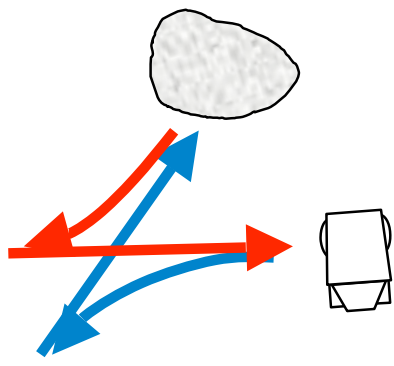

Figure 2: Leaving wing and approach wing

The path planning procedure with the path elements is as follows. At the first step, the curve part is generated. The length of SPC is determined by angle difference between those at the initial point and the final point. Then lengths of straight lines are determined to connect the initial point and the straight part of the approach wing to the both ends of SPC. 
Fig.3 shows a path at an experimental by YZ4. The distance between scooping (or loading) point and switch buck point is about $15 \mathrm{~m}$. It is about two times of the length of the loader. Other problem of this planning method is with narrow reachable area. It causes difficulty on determination of the position of dump truck.

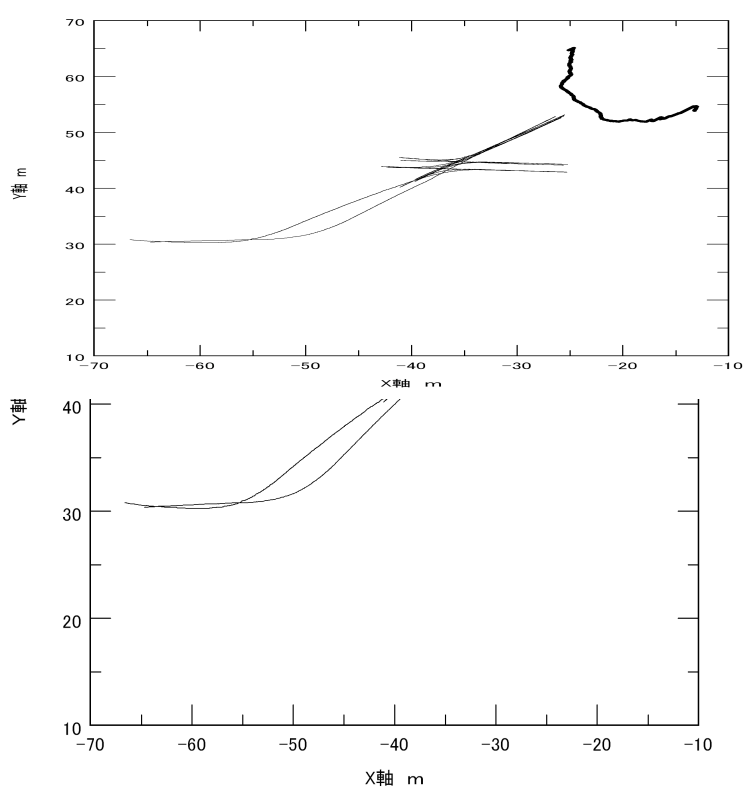

Figure 3: Experimental Result of YZ4

\section{PATH PLANNING WITH LOOK AHEAD METHOD}

Through the observations of loading operation by human operators, some operators control the tip of the bucket on a trajectory which is close to a straight line from the scooping point to the loading point. In these cases, the steering angle at the switch back point is not zero. In the leave wing, the path of the loader is a single curve however the path takes $\mathrm{S}$ shape curve in the approach wing. To achieve these motions, Look Ahead control is applied on the path planning. In this method, the centre of the tip of the bucket is controlled in a direction to the final position.

The steering mechanism of wheel loader is an articulate steering system shown in Fig. 4. The body of the loader is separated into a front part and rear part connected by a centre pin. The angle around the centre pin is controlled by hydraulic cylinders. The placement of the front and rear axles at equal distances from the centre pin ensures that the front and rear wheels travel over the same path, conferring high mobility on off-road surfaces such as mud or soft soil. 


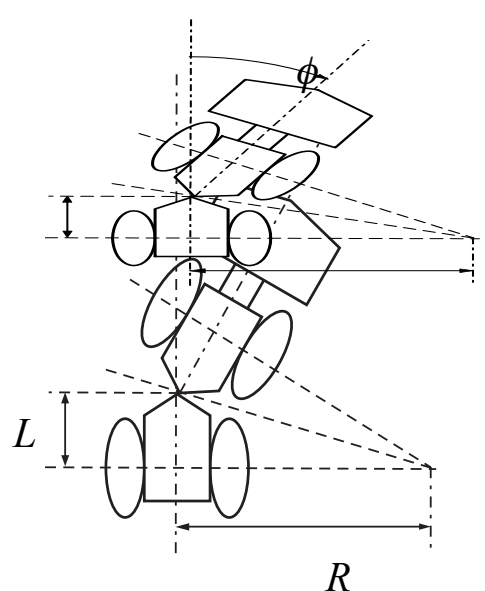

Figure 4: Steering mechanism of wheel loader

Let $\phi$ be the articulate angle, $L$ be the distance from the centre pin to the front or rear axle, $R$ be the radius of curvature, and $\kappa$ be the curvature. The relations between the foregoing are as follows:

$$
\begin{aligned}
& L=R \tan (\phi / 2) \\
& \kappa=1 / R=\frac{2 \tan (\phi / 2)}{L} \\
& \phi=2 \tan (\kappa L / 2)
\end{aligned}
$$

The rotational angle of the front part of body is represented by equation (4). Where $\varpi$ is rotational angle of the body. $\varpi_{s}$ and $\varpi_{d}$ are rotational angles generated by the steering and the advancing of the loader respectively. Each rotational angles are represented by equation (5) and (6) respectively

$$
\begin{aligned}
& \varpi=\varpi_{s}+\varpi_{d} \\
& \varpi_{s}=\dot{\phi} / 2 \\
& \varpi_{d}=\frac{1}{L} \tan \left(\frac{\phi}{2}\right) v
\end{aligned}
$$

Let $\boldsymbol{b}$ be a velocity vector at the centre of tip of the bucket. The elements along $x$ and $y$ axis of a coordinate system fixed on the front part of the body are

$$
\begin{aligned}
& \dot{b}_{x}=v \\
& \dot{b}_{y}=B \varpi=B\left[\frac{\dot{\phi}}{2}+\frac{1}{L} \tan \left(\frac{\phi}{2}\right) v\right]
\end{aligned}
$$


Where $B$ is distance between the centre pin and the tip of the bucket, and $v$ is advancing velocity of the body by wheel revolution (Fig.5).

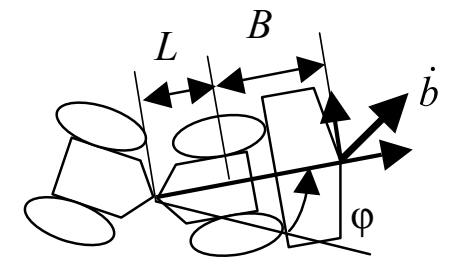

Figure 5: Vector on the tip of the bucket

If the vector $\boldsymbol{b}$ is given, corresponding advancing velocity $v$ and the steering angle velocity $\dot{\phi}$ are obtained by following equations.

$$
\begin{aligned}
& v=\dot{b}_{x} \\
& \dot{\phi}=2\left[\frac{\dot{b}_{y}}{B}-\frac{1}{L} \tan \left(\frac{\phi}{2}\right) v\right]
\end{aligned}
$$

A results of a simulation based on these equations is shown in Fig.6. In the simulation, $L$ : the distance between the centre pin and the front or rear axle is set at $1.25 \mathrm{~m}$ and $B$ : distance between the centre pin and the tip of the bucket is $3 \mathrm{~m}$. Thin line curve represents the tip of the bucket and bold curve represents the centre of the body. The bucket moves to the direction of vector $b$ which points to the final point from the present position. The initial position is $[3,0]$ and the final position is $[4,4]$.

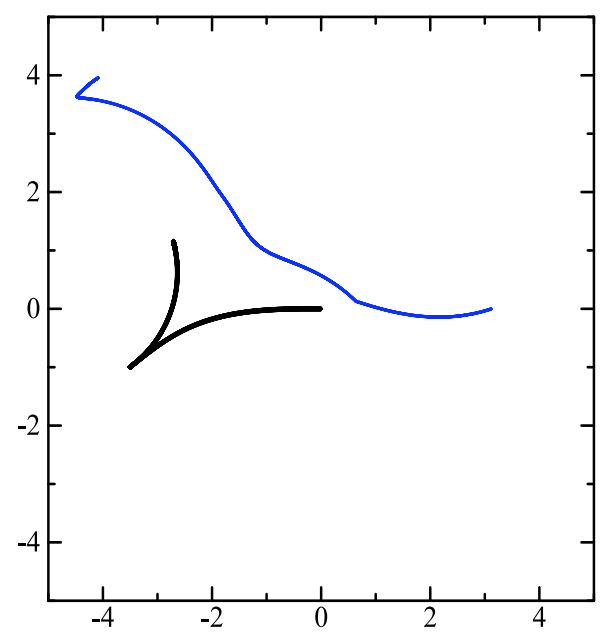

Figure 5: Result of simulation (Look Ahead control)

In this simulation, limits are set on angle of steering at 45 degrees and on the steering angler velocity at 15 degrees/sec. These values are based on performance of the real size experimental model. The tip of the bucket does not exactly move on the straight line from the 
initial point to the final point caused by the limitations on the steering angle however it moves on very short curve connecting the both end points. The result shows that the travelling distance of the loader is very short. But in area near to the switch back point, direction vector $\mathrm{b}$ has large $\mathrm{y}$-axis element compare to $\mathrm{x}$-axis element. The steering angle is changed without wheel revolution. The loader takes "pivoting" motion. It causes undesirable stress on the steering mechanism of the loader.

The most significant disadvantage of Look Ahead control is that the direction of the body and the steering angle at the final point are not controlled because only the position of the tip of the bucket is controlled on this method. For path planning for loading operation, other method should be combined to this method.

\section{COMBINATION OF PATH ELEMENTS AND LOOK AHEAD METHOD}

In the most of operation by human operators, steering angle is not zero at the switch back point on $\mathrm{V}$ shape path. Therefore the leave wing of $\mathrm{V}$ shape path takes simple curve and the approach path takes S shape path. One of the reasons of longer path with SPC is that curvature of the path reduces at the end of SPC. It causes rotational angler velocity reduction at the end part of SPC path. Therefore a longer path is necessary to rotate the body to a setting angle.

In the new developed method, a leaving wing consists of single Pseudo Clothid (PC) and a circle. This combination eliminates the disadvantage mentioned previously. The first part of the path in a leaving wing is PC. The steering angle is increased up to a setting angle then the steering angle is fixed. The second part of the path is a circle which continues until the direction of the loader reaches a setting angle. The steering angle at the end of PC is set at 30 degrees. The angle of the direction of the loader at the end of a circle is set at 45 degrees from the direction at the initial position. These angles are determined based on observation of operations by experienced operators. In the approach wing, Look Ahead method is applied. In Look Ahead method, the simulation results are resembled to straight line following control and the steering angle is almost zero at the end point at certain conditions of the steering angle at the initial point and the distance to the end position.

Fig.6 shows the results of a path planning from the initial point $(0,0)$ to the final point $(10,0)$. The direction of the loader is changed from -20 to 30 degrees at the initial point. The steering angle at the initial point is set at 30 degrees in all cases. Thin curve represents the path of the tip of bucket and bold line represents the centre of the loader. In all cases, the tip of the bucket reaches the final point with small steering angle from 0.2 to 3.8 degree. These small steering angles have almost no effect on the performance of loading or scooping operation. 


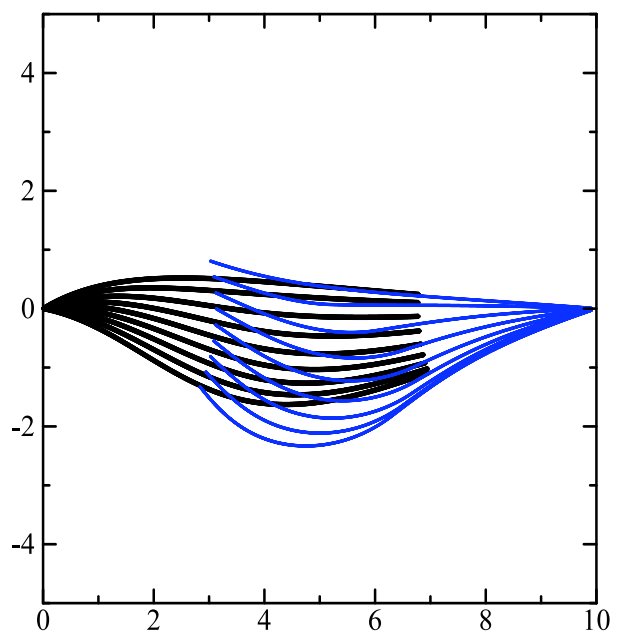

Figure6: Result of planning (different direction)

Fig.7 shows other set of the results of a path planning. The position of the loader at the initial point is changed in $y$ axis direction from $-1 \mathrm{~m}$ to $7 \mathrm{~m}$. The direction of the loader is set at -20 on the initial point. The position of the final point is $(10,0)$ and the steering angle at the initial point is set at 30 degrees same as the previous results in Fig.6. In all cases, the tip of the bucket reaches the final point with small steering angle from 0.0 to 2.4 degree.

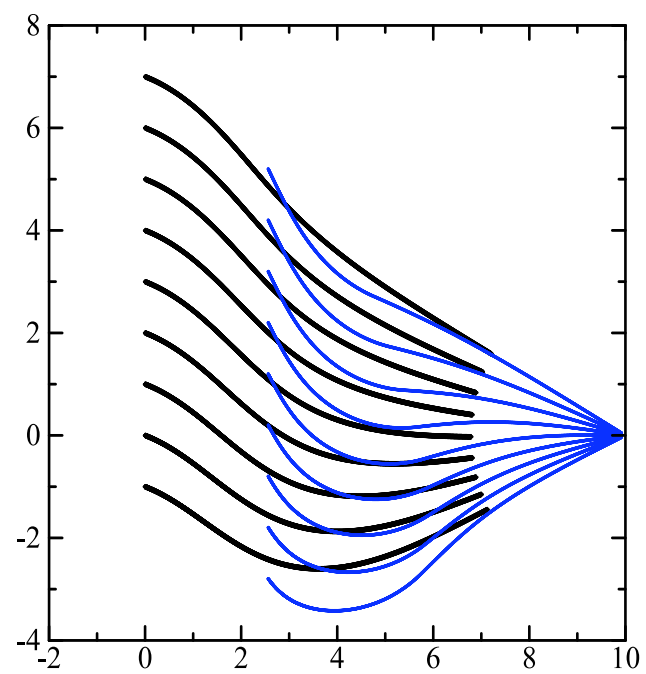

Figure7: Result of planning (different position)

These two results show that this combination of methods makes path planning with flexibility for wide requirements on the path. The loader can reach the final points within these ranges of position and direction, with proper configurations for loading or scooping operation.

Usually, four to six cycles of loading are necessary for filling up the vessel of a dump truck in loading operation. Every loading position should be changed for even and flat loading the material on the vessel. The loading positions of one set of loading for a dump truck have to cover the area of the vessel of the dump truck. Fig. 8 shows the result of planning of V shape path with different final positions to cover a area of the vessel. Adequate paths are planned 
from same switch back point to the different final points. At the every final point, the steering angles are small sufficiently.

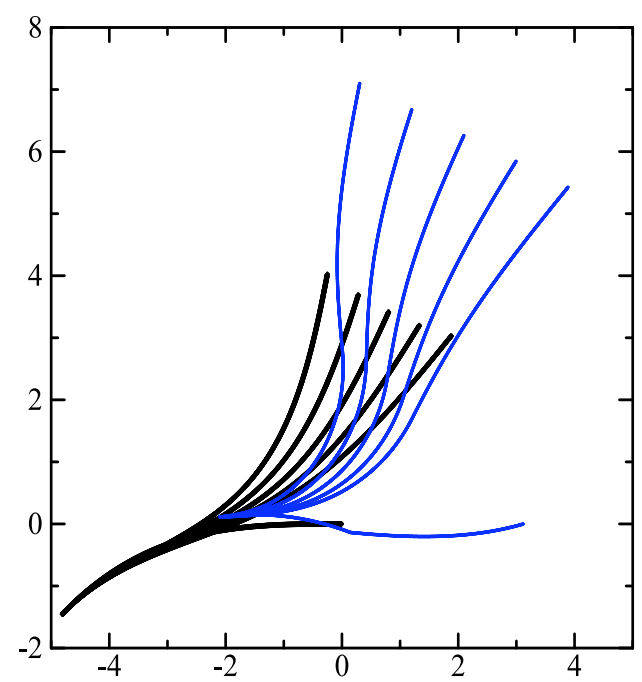

Figure8: Result of planning of $\mathrm{V}$ shape (different final position)

\section{CONCLUSIONS}

New path planning method has been developed. The method is based on observations of performance of human operators in loading operation of wheel loader in surface mines. Planned path consists of combination of path element in the leaving wing of V shape path. In the approach wing, the path is formed based on Look Ahead control method. The planned paths resemble those of operations by human operators. The results of path planning show that the planned path is shorter than that by previous version of the path planning. The developed method has high flexibility to the condition of working place. It generates adequate paths from the same initial point to the various final positions. These final points cover the area of vessel of the dump truck. The developed method will be applied on the real size experimental model for evaluation.

\section{REFERENCES}

[1] Sarata,S. et al.: "Field Test of Autonomous Loading Operation by Wheel Loader", Proc. of IEEE/RSJ International Conference on Intelligent Robots and Systems, pp.2661-2666, 2008 . 